\title{
DESARROLLO, SOSTENIBILIDAD Y CAPACIDADES Una trilogía indesligable
}

\author{
David Solano \\ Universidad ESAN \\ dsolano@esan.edu.pe \\ dadadi96@gmail.com
}

\begin{abstract}
Resumen
La discusión sobre la diferencia entre crecimiento económico y desarrollo es de larga data. Los economistas clásicos se planteaban que lo principal para un país era crear riqueza. Pero con el correr de los años surgieron otros conceptos importantes y complementarios como desarrollo, desarrollo sostenible y capital social, los cuales resaltan la necesidad de capacidades (humanas, institucionales y naturales) para generar bienestar duradero. Las capacidades no acompañan por añadidura a una inversión, hay que trabajar para forjarlas. Pero algunas manifestaciones muestran que muchos tomadores de decisiones públicos y privados en el Perú no son conscientes de esta necesidad; suelen dar prioridad a las capacidades económicas y descuidan las sociales, las ambientales y las institucionales. Sin generación de capacidades no habrá desarrollo posible; y solo con capacidades adecuadas se podrá generar un desarrollo sostenible.
\end{abstract}

Palabras clave: desarrollo, desarrollo sostenible, generación de capacidades.

\begin{abstract}
The difference between economic growth and development has been debated for long. Classical economists hold that the most important challenge for a country was to create wealth. However, as time goes by, other important and complementary concepts have arisen such as development, sustainable development and social capital, stressing out the need for creating human, institutional and natural capacities to generate lasting well-being. Capacities do not come besides investment; we need to create them. However, some examples show that many public and private decision-makers in Peru do not take into account such need; they prioritize economic capacities and neglect social, environmental and institutional ones. Development is not be possible without capacity building, and once the proper capacities are built may sustainable development be created.
\end{abstract}

Key words: development, sustainable development, capacity building. 


\section{Introducción}

Es alarmante la confusión entre los tomadores de decisiones cuando se refieren al desarrollo. No es poco común escucharlos a través de los medios de comunicación declarar «la empresa X traerá desarrollo» o «la carretera $\mathrm{Z}$ generará desarrollo en la zona tal». Aunque esto parezca anecdótico, estas palabras solo expresan la convicción de que una inversión por sí misma traerá desarrollo, evidenciando una visión utilitarista de este y la creencia en que se puede medir en soles, dólares, ladrillos, puentes, aulas o computadoras.

Sucede así porque se considera que crecimiento económico es sinónimo de desarrollo. Hace más de 25 años que los economistas llegaron a la conclusión de que no es así, pues desarrollo económico es crecimiento más una mejor calidad de vida. Esto no solo significa mejor infraestructura o mayor cantidad de dinero circulante, sino también mejor educación, mejores condiciones de salud y empleo, mejores oportunidades de desarrollo personal y profesional y para invertir o realizar negocios, mejor calidad ambiental, instituciones que conocen su razón de ser y son útiles al ciudadano, etc. En resumen, significa generar capacidades. Ninguno de estos aspectos es material ni nace espontáneamente, hay que trabajar en ellos. Por esta razón ninguna inversión por sí sola puede traer desarrollo.

El propósito de este artículo es generar una discusión sobre estos temas, ilustrando aquello que ocasiona esta confusión y cuáles son sus consecuencias. Se debe precisar que la mención a las teorías del desarrollo no tiene la intención de realizar un análisis sistemático y exhaustivo de ellas, sino mostrar brevemente sus ten- dencias y la aparición en su interior del concepto de capacidades. Asimismo, se busca interpretar cómo, en la práctica, estas confusiones conceptuales afectan la toma de decisiones sobre el desarrollo. Y también se plantea una propuesta de utilización de los conceptos para reorientar los procesos de desarrollo hacia su sostenibilidad.

\section{Qué es el desarrollo y cómo se diferencia del crecimiento}

La confusión entre crecimiento y desarrollo no es nueva. Ha sido parte de la discusión sobre las políticas de desarrollo desde que estas existen. Sin embargo, se debe reconocer que la discusión sobre «cómo» debe ser el desarrollo proviene de las ciencias económicas; por tanto, tiene un fuerte componente económico que privilegia la riqueza material como indicador del desarrollo.

En los primeros escritos de los autores clásicos no se mencionaba la palabra «desarrollo», se hablaba de «riqueza» para referirse a los estados de progreso de un país y de los individuos; es decir, la ecuación «desarrollo igual a riqueza material» viene desde los economistas clásicos. Ricardo, en su obra Principios de economía política y tributación, afirma al referirse a los individuos (lo que contenía una definición implícita de pobreza): «Un hombre es rico o pobre, de acuerdo con la abundancia de artículos necesarios y de lujo de que puede disponer; además contribuirán estos artículos en forma igual a la satisfacción de su poseedor» (1959: 126).

En la misma época, Malthus (17661834) decía que una nación es más rica o más pobre en función de lo que tiene para repartir entre sus habitantes; por esto, 
mientras más población tenga más pobre será una sociedad, dado que la provisión de recursos no crece en la misma proporción. Por su parte, Marx (1818-1883), discípulo de Ricardo, señala que las relaciones de producción material determinan la estructura de la sociedad toda. Si bien es cierto Ricardo ya habla de una relación valor-trabajo, Marx indica que la diferencia entre el valor de la mercancía y el trabajo que contiene da lugar a una plusvalía de la cual se apropian los dueños de los medios de producción, lo que genera las desigualdades económicas de una sociedad. Más aún si los que no poseen esos medios de producción se ven sujetos a aceptar los sueldos fijados por la clase dominante o pasar a formar parte de lo que Marx llama «ejército industrial de reserva». Se puede concluir que Marx afirmaba que mientras más rica sea una nación, mayores brechas sociales existirán a su interior.

Por su parte Schumpeter (1883-1950) postula que son los mercados los que dan origen a la riqueza de un país y a su desarrollo. Aun más, una parte importante de su pensamiento es la dicotomía entre economía al servicio de los individuos y de la sociedad y la afirmación de que esto último es propio de las sociedades comunistas. Por otro lado, nuestro compatriota Hernando de Soto afirma que es el capital «la fuerza que eleva la productividad del trabajo y crea la riqueza de las naciones» (2000: 31). Señala que son cinco los aspectos, que llama «misterios del capital», que deben conocerse para poder incrementar el capital de una nación y, por ende, trabajar en su desarrollo:

- Información

- Situación del capital y los activos

- Conciencia política
- Aprender las lecciones de la economía de Estados Unidos

- Leyes y ordenamiento legal

Si bien menciona a los individuos como parte de este entramado, lo hace solo como parte de un ordenamiento establecido que es necesario para el desarrollo.

En resumen, se puede afirmar que estos pensadores conciben el desarrollo como acumulación de riqueza $\mathrm{y}$, como señalan Perroux (1984) y Todaro (1988), la capacidad de incrementar el producto bruto interno (PBI), con la convicción de que la producción por sí misma va a generar desarrollo y que bastan los recursos económicos (o el capital) para que una población sea considerada desarrollada o esté en camino de serlo.

\section{De crecimiento a desarrollo: el papel de las capacidades}

Sin embargo, el crecimiento del PBI como medida de desarrollo ha sido siempre discutible. La gran pregunta que se han hecho los teóricos del desarrollo es cómo medir la satisfacción de las necesidades y cómo el crecimiento económico se refleja en la reducción de la pobreza.

Así, autores como Perroux (1984) plantean la diferencia entre crecimiento y desarrollo. Este menciona que el desarrollo involucra cambios cualitativos, no solo cuantitativos. Desarrollo no es únicamente acumulación de capital, mayor productividad del trabajo y progreso tecnológico, sino también generación de una estructura productiva en la cual las partes (individuos e instituciones) que la constituyen interactúen y se fortalezcan en sus capacidades y habilidades de formación y capacitación. 
Todaro (1988) define el desarrollo como un proceso multidimensional compuesto por grandes transformaciones de las estructuras sociales, las actitudes de la gente y las instituciones nacionales, así como por la aceleración del crecimiento económico, la reducción de la desigualdad y la erradicación de la pobreza absoluta.

Esta necesidad de dotar a los individuos de las herramientas que requieren para generar su propio desarrollo es mencionada inclusive por Adam Smith en La riqueza de las naciones. Él señalaba que el Estado tenía un doble papel, con los individuos y la sociedad:

Dos objetos son los que presenta la economía política, considerada como uno de los ramos de la ciencia de un legislador y que debe cultivar un estadista: el primero [...] habilitar a sus individuos y ponerles en estado de poder surtirse por sí mismos de todo lo necesario; y el segundo, proveer al Estado o República de rentas suficientes para los servicios públicos y las expensas o gastos comunes, dirigiéndose en ambos objetos a enriquecer al Soberano y al pueblo como tales (1997: 45, el subrayado es nuestro).

Schumacher es otro autor que reivindica el papel de las capacidades humanas en la generación del desarrollo:

Toda la historia (como toda la experiencia) apunta al hecho de que es el hombre y no la naturaleza quien proporciona los recursos primarios, que el factor clave de todo desarrollo económico proviene de la mente del hombre. De repente, hay una explosión de coraje, de iniciativa, de invención, de actividad constructiva, no en un solo campo, sino en muchos campos a la misma vez (1978: 56).
Morin (1999) plantea la necesidad de generar conciencia sobre el papel en toda sociedad de las capacidades humanas en la búsqueda de su desarrollo. Señala que son siete los saberes (capacidades) que toda la sociedad debe interiorizar en la búsqueda de su desarrollo:

- Considerar los conocimientos como con defectos y virtudes

- Generar y buscar el conocimiento pertinente para las diferentes situaciones de desarrollo

- Entender a los seres humanos y sus motivaciones

- Entender las limitaciones naturales del crecimiento

- Enfrentar y prepararse para los riesgos

- Prepararse para la convivencia armoniosa con otros

- Proceder con ética

Quizá es Amartya Sen (1998), uno de los más importantes economistas de nuestros tiempos, quien ha sido el abanderado de la reivindicación de la necesidad de dotar a las personas de las capacidades necesarias para poder generar su propio desarrollo. Sen define capacidades como combinaciones alternativas que una persona puede hacer o ser: los distintos funcionamientos que puede lograr. Menciona que los enfoques del desarrollo basados en la acumulación de capital adolecen de varios defectos, entre los cuales están:

- El desinterés hacia el bienestar y la calidad de vida presente y futura. No considerar la trascendencia de los recursos humanos, la relación entre productividad y educación, la atención en salud, alimentación y otros aspectos similares.

- Algunos efectos de la mejora social se reflejan no solo en la productividad 
económica, sino en la mejora del bienestar directo de las personas.

En palabras de Sen:

...la ampliación de la capacidad del ser humano tiene importancia directa e indirecta para conseguir el desarrollo. Indirectamente, permite estimular la productividad, elevar el crecimiento económico, ampliar las prioridades del desarrollo y contribuir a controlar razonablemente el cambio demográfico; directamente, afecta el ámbito de las libertades humanas, del bienestar social y de la calidad de vida, tanto por su valor intrínseco como por su condición de elemento constitutivo de este ámbito (1998: 98).

Sen pone énfasis explícito en las capacidades personales, pero existen también las llamadas capacidades institucionales. El Fondo Monetario Internacional (FMI) define «capacidad institucional» como la capacidad administrativa y de gestión de un país, sobre todo respecto de la aplicación de las políticas económicas, y considera como capacidades necesarias para la lucha contra la pobreza de un país las siguientes:

- Capacidad para recopilar la información estadística necesaria para aplicar la política con eficacia y observando las normas aceptadas internacionalmente.

- Capacidad para planificar con eficacia el gasto público y el suministro de servicios públicos por parte del gobierno central y el gobierno local.

- Capacidad de absorción de la ayuda y de implementación de los proyectos por parte del sector público.

- Eficacia de los organismos en la lucha contra la corrupción y la mejora del gobierno.
- Establecimiento y operación de marcos regulatorios y/o de prudencia adecuados para las empresas y los bancos.

- Adopción y cumplimiento de las reglas y las leyes, así como de las reformas judiciales.

- Protección de los derechos de la propiedad.

- Fomento de la competencia y del sistema económico de mercado en general.

Resulta llamativo que en esta definición del FMI no se haga mención a la necesaria capacidad de desarrollo de las personas, pues se trata de un enfoque eminentemente tecnocrático y legal. Pero es importante tenerlo en cuenta pues este concepto es el que está presente en casi todas las definiciones y las acciones de desarrollo de los países.

Paralelamente, en la década de 1980 se vislumbraron los primeros albores de otro concepto ligado estrechamente a las capacidades humanas. Este es el concepto de capital social, el cual se ha ido refinando hasta nuestros días y aún está sujeto a debates conceptuales ${ }^{1}$. Se define como: «....aspectos de la organización social tales como confianza, normas y redes, que pueden mejorar la eficiencia de una sociedad al facilitar la acción coordinada» (Putnam, 1993: 167); o, como afirman Robison et al. (2000), la simpatía de una persona o un grupo hacia otra persona o grupo que puede producir un beneficio potencial, una ventaja y un tratamiento preferencial para otra persona

1. Es necesario mencionar que en algunas zonas y organizaciones del Perú existe resistencia a usar el término «capital social» porque se afirma que reduce a las personas a medios de producción. Esta discusión no será profundizada en esta ocasión. 
o grupo de personas más allá del esperado en una relación de intercambio. Es decir, el concepto de capital social alude a las capacidades de un grupo de formar redes y apoyarse mutuamente, sea en aspectos económicos, sociales, culturales, laborales, etc., con el fin de buscar su desarrollo.

En esa misma década surge otro concepto también vinculado a las capacidades. Esta vez ligado a las capacidades naturales de los ecosistemas del planeta para soportar el crecimiento económico. Y es el concepto de desarrollo sostenible. La Comisión Brudtland (1987) o Comisión Mundial sobre el Medio Ambiente y el Desarrollo (CMMAD), coordinada por la primera ministra de Noruega, Gro Harlem Brudtland, publicó el informe titulado Nuestro futuro común, también conocido como Informe Brudtland. Este incluyó el tema ambiental y de los recursos naturales en la discusión del desarrollo, acuñando el concepto de desarrollo sostenible: aquel que satisface las necesidades del presente sin comprometer el potencial de satisfacer las necesidades de las generaciones futuras. Este enfoque sugiere que el desarrollo económico (en verdad, el crecimiento económico, en un sentido conceptual exacto) ha subvalorado las bases ambientales y sociales del desarrollo, haciéndolo poco duradero.

Por su parte, el Programa de Naciones Unidas para el Desarrollo (PNUD) introduce, en 1992, el concepto de desarrollo humano: «...el proceso de ampliar la gama de opciones de las personas brindándoles mayores oportunidades de educación, atención médica, ingreso y empleo abarcando el espectro total de opciones humanas, desde un entorno físico en buenas condiciones hasta libertades políticas y económicas» (PNUD, 2007).
Ya en este siglo, la Declaración de las Naciones Unidas, firmada por los países miembros en septiembre de 2000 donde se plantean ocho objetivos que deben lograrse en el año 2015, da lugar a los denominados Objetivos del Milenio. Estos objetivos constituyen por sí mismos un concepto sobre desarrollo sostenible:

- Objetivo 1: Erradicar la pobreza extrema y el hambre.

- Objetivo 2: Lograr la enseñanza primaria universal.

- Objetivo 3: Promover la igualdad entre géneros y la autonomía de la mujer.

- Objetivo 4: Reducir la mortalidad infantil.

- Objetivo 5: Mejorar la salud materna.

- Objetivo 6: Combatir el VIH/sida, el paludismo y otras enfermedades.

- Objetivo 7: Garantizar la sostenibilidad del medio ambiente.

- Objetivo 8: Fomentar una asociación mundial para el desarrollo.

\section{Los cuatro ejes del desarrollo}

Se puede concluir que el enfoque de desarrollo univariable (solo económico) es un concepto anacrónico. Si se analiza los aspectos comunes y distintos de estos conceptos se puede afirmar que el desarrollo comprende cuatro aspectos fundamentales:

- Económico. Como se ha visto, la discusión sobre el desarrollo se generó desde el ámbito económico. Actualmente no se discute que no podrá existir desarrollo sin recursos económicos, sin inversiones. Sin estos, no se podrá 
financiar el desarrollo ni generar la necesaria base material del desarrollo, como la infraestructura o el equipamiento.

- Social. Para generar desarrollo es necesario lograr que la población se incorpore y haga suyos los procesos de desarrollo. Sin embargo, en la práctica, estos esfuerzos se han circunscrito a los temas materiales (como hospitales o escuelas) cuando existe la necesidad de generar capacidades intrínsecas a las personas como son la educación o la salud y otras condiciones de integración a la sociedad.

- Ambiental. La calidad y el uso sostenible de los recursos son una condición necesaria para el desarrollo. No puede concebirse como desarrollada una sociedad que ha perdido o está en proceso de pérdida de su capital natural en aras de crear condiciones económicas temporales. La historia del Perú muestra algunos ejemplos antiguos (el guano de las islas o el caucho, a fines del siglo XIX) o recientes (el boom pesquero de la década de 1970 en la costa del Perú) en los cuales, por generar condiciones económicas, se perdió la calidad del ambiente y se deterioraron o agotaron los recursos naturales, lo que provocó condiciones de pobreza en las zonas escenario de estas actividades.

- Institucional. Mencionado explícitamente por Todaro y tomado por la Cepal en el llamado «sistema socio-ecológico» (Gallopin, 2006), el cual reúne las estructuras nacionales, las leyes y las estructuras de poder. Las definiciones presentadas incluyen este tema, en forma implícita o explícita.
Para todos estos aspectos es necesario desarrollar capacidades. Aunque en los párrafos anteriores las capacidades solo se hacen explícitas en el tema social, los individuos necesitan capacidades para abordar los cuatro ejes del desarrollo. Requieren capacidades para su desarrollo económico, social, ambiental e institucional. Todos estos procesos son desarrollados por personas, y son las personas las que necesitan no solo conocer conceptos, sino también «saber cómo» generar estos desarrollos.

Cabe preguntarse ¿cómo se espera que los individuos se incorporen a los procesos de desarrollo ${ }^{2}$ si no cuentan con las capacidades (conocimientos, actitudes y/o destrezas técnicas) para poder participar activamente en aquellos en marcha? Cómo se espera que un poblador rural sea parte de los procesos de desarrollo si no conoce a ciencia cierta el beneficio o el perjuicio de una determinada inversión en su vida diaria, y si no sabe cómo ser parte de este progreso, sea directamente laborando para la empresa inversionista o indirectamente como parte de la cadena comercial o de servicios que provee a esta empresa y sus actividades conexas. Por ejemplo, cómo un campesino puede beneficiarse económicamente de una inversión minera en su lugar de residencia si no tiene las capacidades técnicas para trabajar en la empresa (y no tiene esperanzas de que sus hijos las adquieran) o no sabe cómo proveer alimentos o servicios a esta empresa.

También se debe cuestionar cómo se espera que exista un desarrollo que perdure en un pueblo si por cada sol que recibe debe

2. En adelante se define como proceso de desarrollo toda acción, proyecto o programa que busca generar resultados en temas económicos, sociales, ambientales $\mathrm{y} / \mathrm{o}$ institucionales. 
invertir dos para curar su salud deteriorada por los procesos de contaminación. Es un contrasentido, por ejemplo, tener un Estado que gasta millones de soles en atenciones de salud y, por otro lado, permite (por acción equivocada o inacción) niveles de contaminación que precisamente incrementan la morbilidad. O tener pobladores que deben invertir sus fuerzas y tiempo en cuidar recursos naturales que consideran suyos para que no sean utilizados por otros sin obtener beneficios para ellos, o para que en su explotación no se afecte su calidad de vida como consecuencia de la contaminación.

Asimismo, es importante reflexionar sobre cómo se va a sostener un proceso de desarrollo si las instituciones no tienen las competencias técnicas, legales o presupuestales para poder intervenir ayudando a generar desarrollo o dando servicios al ciudadano para que participe en este proceso.

Como se puede ver, la generación del desarrollo va más allá de ser un tema económico, del capital, o de dar asistencia a las personas que viven en la pobreza. Si se toma en cuenta que esta no es solo la ausencia de bienes materiales, como se pensaba a principios del siglo XX (Rowntree, 1901), y se considera que pobreza también es la escasez de capacidades para ser mejores y progresar, se puede señalar como primera conclusión que (y no hay ejemplo empírico en el mundo que muestre lo contrario) un gobierno que apueste todo sus recursos y capacidades a mejorar solo las inversiones y dar asistencia económica, alimentaria y de infraestructura a los pobladores jamás podrá conseguir el desarrollo si no busca generar capacidades en las personas receptoras de estos procesos, mejorar su relación con el ambiente donde viven y fortalecer sus instituciones.
Asumiendo que el desarrollo no es solo económico, las teorías modernas del desarrollo ofrecen propuestas de cómo llegar a él. Entre estas se puede mencionar la teoría de la modernización (el ahorro y la inversión son el motor del desarrollo, base del llamado «chorreo» del progreso a los más pobres); la estructuralista (el desarrollo como producto del intercambio con otros países, el problema es cómo superar la dicotomía de un sector exportador moderno $\mathrm{y}$ un sector atrasado que produce para el mercado interno); la neomarxista (postula el asistencialismo y el cambio social a través de grandes transformaciones); y la neoliberal (siempre habrá desigualdad, lo que actúa como un incentivo del desarrollo humano). Sin embargo, cada vez hay más consenso en América Latina acerca de que el desarrollo debe respetar los cuatro ejes de la sostenibilidad. Por tanto, se puede concluir que el desarrollo si no es sostenible no es desarrollo: no existe desarrollo sin sostenibilidad.

Se debe aceptar que el desarrollo no solo implica el aspecto económico y material. Implica transformaciones en la sociedad privilegiando el respeto a la realidad del otro; conlleva la necesidad de generar las condiciones para que un poblador pueda crecer como persona o trabajador; implica reconocer nuestro papel en la sociedad y cumplirlo; implica respetar el ambiente propio y el de los demás; y también entender que la propia situación económica o social, de abundancia o escasez, no da derecho a pasar encima de los derechos de otros. Todo esto se debe realizar respetando, generando y fortaleciendo las necesarias capacidades humanas para ser partícipes de estos procesos. Solo una sociedad que comprenda estos aspectos y trabaje en ellos está en el verdadero camino hacia su desarrollo. 


\section{4. ¿Los tomadores de decisiones en el Perú son conscientes de estos conceptos de desarrollo?}

La evidencia empírica siempre ha mostrado que «conceptos equivocados llevan a decisiones equivocadas» y el tema del desarrollo no es la excepción. Pero es necesario deslindar el «no ser consciente» de cualquier acepción peyorativa. Según el Consejo Nacional del Ambiente (Conam, 2004), ser consciente significa no solo conocer los conceptos sino también aplicarlos en la práctica.

El Perú y su gobierno tienen como principal reto generar desarrollo. Este reto es más urgente en un escenario como el actual con una tasa de crecimiento del PBI de cerca de $8 \%$ anual, $48 \%$ de pobreza y casi la mitad de una población que votó por un candidato cuya principal bandera era terminar con el sistema democrático actual, por considerar que no favorece al desarrollo humano equilibrado y solo privilegia a algunos. En función de estas evidencias, no se pueden perder cinco años en ese esfuerzo. Por esta razón, la principal tarea del gobierno debe ser trabajar en estos cuatro ejes para lograr un desarrollo que perdure en el tiempo.

Sin embargo, se puede apreciar algunas decisiones y manifestaciones que hacen dudar de que quienes toman las decisiones (públicas y privadas) sean realmente conscientes de las nuevas corrientes y los nuevos conceptos de desarrollo. A continuación se señalan algunas manifestaciones que apoyan esta situación.

a) Privilegio de lo económico sobre los otros tres aspectos. No es poco común escuchar a importantes tomadores de decisiones (sobre todo públicos) men- cionar que aspectos como el ambiental «pueden frenar el desarrollo y ahuyentar inversiones». Basta citar al presidente Alan García quien afirma: «...el viejo comunista anticapitalista del siglo XIX se disfrazó de proteccionista en el siglo XX y cambia otra vez de camiseta en el siglo XXI para ser medioambientalista. Pero siempre anticapitalista, contra la inversión, sin explicar cómo, con una agricultura pobre, se podría dar un salto a un mayor desarrollo» (2007). Esta generalización se puede leer como un pensamiento que pone el aspecto económico por encima de los otros tres ejes de la sostenibilidad. Sin embargo, está demostrado que las mejores inversiones para el desarrollo integral del país son aquellas que toman en cuenta todos estos ejes al momento de intervenir en un espacio.

b) La población no se siente parte de los procesos de desarrollo. Los países y las regiones apuestan por procesos de desarrollo en función de sus ventajas competitivas. El Perú, por ejemplo, en la práctica ha apostado sobre todo por un modelo de desarrollo que busca generar las condiciones necesarias para que los inversionistas apuesten por el Perú en diferentes ramas, pero la más destacada es la minería. Sin embargo, esta situación ha dado lugar a diversas manifestaciones de rechazo de la población ante estas inversiones. Esto no es más que el reflejo de una población que no se siente preparada para intervenir en estos procesos y es fácil presa de algunas ideas contrarias a esta opción de desarrollo. Las deficiencias conceptuales se vislumbran cuando se busca atacar solo los síntomas (las protestas) y no las causas (cómo generar capacidades conceptuales y destrezas 
para que la población se involucre en estos procesos, sea en la oferta de trabajo, bienes o servicios). Esta es una deficiencia que se percibe tanto en el sector público como en el privado y está relacionada con el siguiente aspecto.

c) Reducción de lo social a lo asistencial. Se considera que para atender las necesidades sociales se necesita infraestructura o dinero. Y los pobladores, incluidas sus autoridades, estiman que una negociación con una empresa se debe plantear en términos de miles de soles más o menos, sin pensar para qué. Pero este dinero es muy pocas veces invertido en generar capacidades e, inclusive cuando esto se hace, se limita a ofrecer cursos de capacitación. Construir infraestructura también puede ser considerado generar capacidades, pero solo cuando esta es parte de una propuesta sostenible de trabajo. Es decir, la infraestructura, el asistencialismo o la capacitación deben ser medios para lograr un objetivo mayor, y no fines en sí mismos. En este sentido se puede citar como ejemplo la distribución de los fondos mineros creados por el Decreto Supremo 071-2006-EM, los cuales, según la Sociedad de Minería, Petróleo y Energía, la inversión del año 2007 en «Fortalecimiento de capacidades» es apenas el 5\% de la inversión total.

\section{d) Escasa prioridad política a lo ambien-} tal. Por las razones expuestas anteriormente, se considera lo ambiental como un tema anexo. Si bien el discurso ha cambiado en los últimos años y ahora se integra lo ambiental como parte de «lo que se debe hacer», no se percibe aún como condición sine qua non del desarrollo. Dado que un indicador de prioridad es la asignación de presupuesto, se puede citar como muestra el monto del presupuesto del Conam, la autoridad ambiental nacional, el cual es de 17 millones de soles para el año fiscal 2008 (con un aumento de 70\% en relación a lo asignado en promedio en los últimos cinco años); en contraste con lo asignado a las cabezas de los otros ejes del desarrollo como el Ministerio de Economía y Finanzas (13 mil millones de soles) o el Ministerio de la Mujer y el Desarrollo Humano (350 millones de soles). Tampoco se compara con el monto del que dispone el Ministerio de Educación (3 mil millones de soles) o algunos municipios como San Marcos en Áncash (139 millones de soles) o Echarate en Cusco (121 millones de soles). También se puede tomar como indicador la ausencia del Conam en las decisiones acerca del desarrollo nacional que se toman en el Consejo de Ministros. O el que los sectores económicos tengan autonomía al tomar decisiones, sean o no estas contrarias al uso sostenible del ambiente y los recursos naturales ${ }^{3}$.

3. Una situación «anecdótica» que muestra la escasa prioridad política del tema ambiental se presentó en la primera mitad del año 2003. La autógrafa de la Ley del Sistema Nacional de Gestión Ambiental, aprobada por el Congreso, fue observada por el Poder Ejecutivo. Una de las razones de esta observación fue que otorgaba al Conam el rango de ministerio y a su presidente un lugar en el Consejo de Ministros, pues ya no se permitía la figura de «rango de Ministerio» por razones presupuestales. Sin embargo, en julio de ese mismo año, el presidente de la República anunció en su discurso de Fiestas Patrias que el Instituto Peruano del Deporte había sido elevado al rango de ministerio. 
e) Escasez de capacidades humanas en el sector público. Situación que se repite gobierno tras gobierno. Es una excelente estrategia para obtener la aprobación popular pagar sueldos bajos a los empleados públicos, considerados tradicionalmente incompetentes y «ociosos». En países como el Perú, a través de la historia, los puestos públicos han sido botín político de los distintos gobiernos. Sin embargo, es importante mencionar que el empleado público debe ser el enlace entre la población y las instituciones para generar desarrollo, es parte importante del eje institucional del desarrollo. El que no haya cumplido esta función no significa que el perfil del empleado público deba ser de baja calidad profesional. La realidad muestra que los empleados públicos están dedicados a hacer labores rutinarias y no se han convertido en verdaderos impulsores de desarrollo, en algunos casos por no contar con la capacidad para hacerlo o por el exceso de controles que la propia actividad pública impone. Este desconocimiento del papel de las instituciones en el desarrollo hace que la función pública se haya desvalorado y se considera como «una gran decisión» contar con un sector público subremunerado.

f) Desconocimiento del verdadero papel de la educación en el desarrollo. En una construcción del desarrollo que se basa en la construcción de capacidades humanas, el sector educación se convierte en eje fundamental de esta propuesta. La educación no se refiere solo a lo formal (en sus diversos niveles y modalidades) sino también lo no formal (que se da en la sociedad). Sin embargo, ilustra este problema el hecho de que el sector educación no cuenta con una estrategia acerca de las capacidades que debe formar en el ciudadano peruano (que es su verdadera función), sino únicamente con un Diseño Curricular Nacional que resume las capacidades que deben formarse en la etapa escolar. No obstante, con decisiones que muestran la escasa claridad acerca de las capacidades que deben formarse para el desarrollo, se generan continuos cambios de este diseño (tres veces en el periodo 2001-2006 y se anuncia otro para el año 2009), lo que deja claro que se desconoce la realidad del Perú. Es muy difícil que los maestros (aparte de todos los problemas gremiales que conocemos) puedan dedicarse a formar si también deben aprender las nuevas formas de programación cada dos años. En cuanto a la educación universitaria, no existe un diseño de los profesionales que necesita el país en cada rama. En educación no formal no existe ninguna propuesta acerca de las capacidades que deben fomentarse en el ciudadano peruano para contribuir al desarrollo. En resumen, el sector educación no cumple su papel de ente rector no solo de los procesos educativos formales sino de las capacidades que requiere el ciudadano peruano para aportar en el desarrollo.

\section{g) Considerar que una sola institución} puede asumir algún tema transversal como educación, pobreza o ambiente. Por definición, los temas transversales son importantes para la sociedad y el desarrollo. Los temas transversales son aquellos que deben abordarse para generar el desarrollo sostenible de los pueblos, como las cuestiones sociales, la educación, la lucha contra la pobreza, la protección del ambiente, la ciudadanía, la cuestión de género, los 
derechos humanos, la diversidad cultural, etc. Sin embargo, estos temas no son abordados transversalmente, sino en forma fragmentada, sin una visión común del Estado sobre ellos. Está demostrado que una sola institución no puede encargarse de la educación, la pobreza o el medio ambiente, pues se necesita lograr que todas las instituciones incorporen estos temas como ejes de trabajo.

h) Considerar la participación ciudadana como un requisito legal o una herramienta para solucionar conflictos. Son muy pocas las instituciones que han hecho de la participación "proactiva» (lo contrario de reactiva) un eje de trabajo importante. Lo más común es el caso contrario, instituciones y empresas que otorgan muy poca importancia a la participación y a los procesos de interacción con la población, sea por no valorar sus beneficios o por temor a interactuar con el público; pero sufren las consecuencias de los conflictos generados precisamente por no generar una participación continua. Un indicador de que el tema de la participación se trata en forma reactiva son las numerosas mesas de diálogo que buscan enfrentar conflictos que son presididas por el presidente del Consejo de Ministros y configuran soluciones políticas que surgen cuando las soluciones técnicas han fracasado. Lo cierto es que, en un proceso de desarrollo que necesita generar capacidades, la interacción con el público es fundamental para conocer las necesidades de desarrollo de esa población o identificar posibles conflictos en gestación. Una institución que hace de la participación un eje de trabajo tiene mayores posibilidades de generar un proceso de desarrollo sostenible. En nuestro país se está cometiendo el grave error de desvirtuar la participación y la concertación, convirtiéndolas en herramienta política de última instancia y desligándolas de su verdadero papel de herramienta técnica que debe estar presente en todo el proceso de desarrollo.

Todas estas son muestras visibles de la escasa conciencia sobre el verdadero significado de estos conceptos. Y, definitivamente, se convierten en obstáculos para los procesos de desarrollo.

\section{Capacidades para dar sostenibilidad al desarrollo}

Dados los cuatro ejes que requiere el desarrollo y los retos del Perú, es necesario tener mucha claridad acerca de cuáles serían las capacidades que deben formarse en sus ciudadanos para poder construir un desarrollo verdadero, es decir, las capacidades que se requieren para lograr el desarrollo económico, social, ambiental e institucional que se describen a continuación.

\subsection{Capacidades necesarias para generar desarrollo económico}

- Identificación de oportunidades y generación de propuestas de inversión. Dado que existe un crecimiento económico importante y fondos que deben ser invertidos.

- Planificación de la oportunidad de los flujos adecuados de fondos (en cantidad y oportunidad). No se puede esperar éxito en un proceso de desarrollo si los flujos de fondos no son adecuados al devenir del proceso. Y los problemas con los fondos no se limitan a que sean 
insuficientes, sino que además no puedan cubrir oportunamente las acciones previstas. Muchos proyectos han fracasado a pesar de tener fondos disponibles, solo debido a que los trámites y los procesos administrativos son engorrosos y desaniman a los involucrados. Esto es frecuente en el sector público, donde las dificultades para ejecutar los presupuestos generan muchos obstáculos en los procesos de desarrollo que se inician.

- Metodologías y procedimientos para generar la conservación o el incremento del capital. No es un secreto que una entidad no puede descapitalizarse, pero el capital de un país o una ciudad no solo es el capital económico-financiero, sino también el capital natural (los recursos naturales) y social (las relaciones sociales y las redes de trabajo).

- Certeza de la disponibilidad de activos suficientes y flujos de activos adecuados. Un proceso de desarrollo debe asegurar los activos suficientes para cubrir la máxima demanda. Muchas veces se inician procesos sin considerar el crecimiento de la demanda de intervenciones en el mediano plazo, lo cual hace que haya una demanda insatisfecha o que se dividan los activos existentes entre un público que crece, lo que atenta contra la calidad de los resultados obtenidos.

\subsection{Capacidades necesarias para generar desarrollo social}

- Objetivos comunes de la población. Cuando la población no comparte un objetivo común, es decir, una idea ho- mogénea, aunque sea muy general, de aquello que busca lograr en un proceso de desarrollo será muy difícil que se obtengan resultados importantes. Esto porque cada persona puede tener distintas imágenes-objetivo de su situación esperada al final del proceso de desarrollo, lo cual generará el descontento de aquellos que no se sienten satisfechos y puede hacer fracasar el proceso de búsqueda de resultados significativos para el desarrollo de los pueblos.

- Preparación del público objetivo para responder a los procesos. Es necesario tener muy claro lo que necesita un proceso por parte de la población. Para ello se debe hacer un diagnóstico de las capacidades (conceptos, actitudes y destrezas) con las cuales cuenta la población y, en caso de ser insuficientes, desarrollar procesos previos de generación de capacidades. Muchas veces se comete el error de exigir a la población cierto tipo de apoyo para el que no se encuentra preparada, lo que trae como consecuencia el rechazo y el fracaso de los procesos.

- Participación amplia. No existirá desarrollo de una comunidad si la población no se involucra en el proceso que se genere. Para ello, la educación es una herramienta fundamental.

- Aceptación de los procesos por parte del público. La población objeto del proceso de desarrollo debe aceptarlo como importante para ella, de lo contrario existirán resistencias $\mathrm{y}$, eventualmente, protestas ante su avance, lo cual impedirá continuar en busca de resultados. 
- Claridad sobre las formas en las cuales el público objetivo puede contribuir al proceso de desarrollo. En ocasiones, la población conoce el proceso y lo acepta, pero no tiene claro cómo puede insertarse en él; por tanto, es necesario tener claro el papel de los actores y prepararlos para ello.

- Disponibilidad de canales para permitir la participación. Muchas veces las protestas y el descontento se producen porque las personas sienten que no son escuchadas por los promotores de un proceso de desarrollo. Es necesario generar los mecanismos institucionales (no personales o espontáneos) a través de los cuales las personas involucradas hagan llegar sus aportes o críticas.

- Compromiso de los actores. Hay procesos de desarrollo en los cuales las personas involucradas no se comprometen con estos, a pesar de estar participando. El compromiso significa que las personas y las instituciones están dispuestas a seguir con el proceso una vez terminados los beneficios (económicos o materiales) que pueden estar recibiendo. Para esto se deben brindar beneficios económicos o materiales asegurando una participación real; de no ser así, se atenta claramente contra la sostenibilidad del proceso, pues nada asegura que el trabajo que se realiza continúe una vez terminada la fase inicial.

- Promoción y refuerzo de los liderazgos. Es necesario que los líderes locales estén involucrados en los procesos, sin tratar de menoscabar su liderazgo si piensan diferente o manifiestan su desacuerdo.
- Participación de los disidentes. Está relacionada con el punto anterior, es necesario involucrar a todos los actores importantes, inclusive aquellos que piensan diferente o están en desacuerdo. Estos se pueden convertir en enemigos del proceso y lograr adeptos ante las primeras dificultades, más aún si se sienten rechazados o excluidos del proceso.

\subsection{Capacidades necesarias para generar desarrollo ambiental}

- Uso compartido y consensuado de los recursos escasos. Si un proceso genera disputas por recursos escasos (agua, tierra, etc.) tiene altas probabilidades de no ser sostenible. Esto empeora si se resuelve por imposición legal o económica, pues genera un problema latente que puede surgir nuevamente en cualquier etapa posterior del proceso.

- Crecimiento de los recursos naturales renovables y los servicios en igual o mayor medida que las actividades que se generan. Existen inversiones que demandan recursos naturales y servicios que no se incrementan para cubrir la demanda adicional resultado de los nuevos procesos. Esto puede generar en el futuro disputas por recursos escasos o hacer colapsar los recursos existentes ${ }^{4}$.

4. Este es el caso del proyecto Mi Vivienda que promueve la construcción de viviendas multifamiliares en zonas donde las instalaciones urbanas no están preparadas para el aumento explosivo del número de nuevas familias, ya que en espacios donde antes habitaban cinco o diez familias ahora viven entre 30 y 150 , sin que las instalaciones de agua, desagüe o energía estén preparadas. Esto ya está haciendo colapsar algunas instalaciones, ya que no hay indicios de que los gobiernos locales analicen esta situación antes de otorgar las licencias. 
- Recursos no afectados por sobreuso o contaminación. Se trata de las formas de impacto ambiental más conocidas. El sobreuso de los recursos y la contaminación atentan contra la sostenibilidad de cualquier proceso, pues en el mediano plazo esto va a afectar la salud y la calidad de vida de la población.

- Población no polarizada. La polarización de la población constituye un impacto ambiental importante, pues afecta la convivencia armónica entre empresa y sociedad, aspecto fundamental para vivir en armonía con el medio, objetivo fundamental de la gestión ambiental.

- Mecanismos institucionales suficientes y capaces para trabajar los conflictos. Los conflictos se generan por problemas no resueltos, por ello todo proceso de desarrollo debe abordar oportunamente las situaciones problemáticas. Asimismo, se debe diseñar los mecanismos para abordar los posibles conflictos que surjan. Estos mecanismos son las mesas de diálogo o las comisiones de trabajo. Sin embargo, se debe tener en cuenta que estos mecanismos no resuelven los conflictos, solo permiten el trabajo conjunto entre las partes.

- Gestión adecuada del riesgo. Los procesos de desarrollo deben incorporar la gestión del riesgo de desastres. Deben buscar no solo prepararse para los posibles desastres sino, además, no generar nuevas condiciones de vulnerabilidad de la población y mejorar las condiciones de vulnerabilidad existentes. Un peligro natural puede hacer perder millonarias inversiones en procesos de desarrollo si los promotores no están preparados ante él. Una condición necesaria es generar las condiciones de resiliencia, es decir, la capacidad de la población de recuperarse ante un evento natural desfavorable.

- Respeto de la potencialidad del espacio. Esto significa respetar su capacidad de uso en el largo plazo. Es importante privilegiar los recursos cuyo uso puede continuar en el largo plazo frente a aquellos que tienen corta duración. Lo ideal es poder hacer compatible el uso de ambos tipos de recursos para poder evitar el rechazo de un sector de la población que puede verse marginada del desarrollo.

\subsection{Capacidades necesarias para lograr el desarrollo institucional}

La sostenibilidad de un proceso de desarrollo no está ligada solo a las condiciones externas. También las instituciones que los promueven deben estar preparadas para ello. Esto a pesar de ser lo más cercano y manejable por cualquier institución gestora de procesos de desarrollo es lo menos trabajado en la práctica. Por ello es importante señalar las capacidades que se deben generar para un adecuado desarrollo institucional:

- Organizaciones adecuadas para llevar a cabo el proceso. El ente promotor del proceso de desarrollo debe adaptar su organización interna para ser exitosa en el proceso que emprende. Sucede, sobre todo en el ámbito público, que se pretende que la realidad se adapte a la organización y no al revés, como debe ser. 
- Personal adecuado en calidad y número. Como dice el principio de Peter, todos somos competentes hasta llegar a nuestro nivel de incompetencia. $\mathrm{Mu}-$ chas veces se pretende que las personas sean competentes para todos los procesos, sin analizar si cuentan con las capacidades adecuadas para liderar o ser parte de un proceso de desarrollo determinado. Otro problema es no contar con un equipo con el número suficiente de participantes para atender las demandas de la población objetivo.

- Claridad de la propuesta al interior de la organización que promueve el proceso de desarrollo. Así como los objetivos no comunes de la población impiden lograr objetivos, también si el equipo de trabajo cuenta con una imagen-objetivo diferente y una distinta percepción de los procesos no se podrá lograr resultados sostenibles. Esto privilegia y reivindica la necesidad de los mecanismos de comunicación interna.

- Mensaje similar entre todos los miembros de la institución que promueve el proceso de desarrollo. Si el aspecto anterior trata el aspecto interno, este se ocupa del aspecto externo. Si el público objetivo recibe del equipo de trabajo mensajes diferentes sobre las acciones y los objetivos a lograr, o simplemente el equipo maneja y difunde conceptos diferentes, el público se confundirá e interpretará el trabajo a realizar como mejor le convenga, lo que en algún momento traerá la insatisfacción de algunos grupos.

- Compromiso de la alta dirección con los objetivos. Como toda decisión estratégica, generar un proceso de de- sarrollo requiere un compromiso total de la alta dirección. Muchas veces el trabajo en temas de desarrollo requiere decisiones importantes pues no siempre se cumplen las condiciones previstas, por ello, si la alta dirección está atenta e involucrada es mucho más sencillo resolver esas dificultades y asegurar el compromiso del público objetivo.

- Maquinaria y equipo suficiente. La maquinaria y el equipo también deben ser proyectados para satisfacer las necesidades y las demandas de trabajo.

- Procedimientos claros que guíen la toma de decisiones y la forma de afrontar las diferentes situaciones. Para evitar decisiones diferentes ante situaciones similares, lo que genera confusión en el público objetivo.

- Métodos claros para detectar y corregir desviaciones. En todo proceso hay situaciones que salen de los estándares establecidos. Esto no se puede evitar y la organización debe estar preparada para ello y plantear formas de abordar estas desviaciones en forma institucionalizada para hacer las correcciones que permitan conseguir los objetivos planteados. Se debe tener en cuenta que lo único que no pueden cambiar son los objetivos, las formas pueden adaptarse a las circunstancias.

- Motivación e identificación del personal con la propuesta de desarrollo. El trabajo de desarrollo es fundamentalmente de campo, y si el personal no se siente motivado con el trabajo que hace es muy difícil asegurar la calidad de los resultados. Es imposible controlar al personal en campo, por ello deben sentirse motivados con el trabajo 
que hacen, solo así podrán contribuir al éxito del proceso.

- Áreas diferentes a las que lideran el proceso deben conocer la propuesta y cómo contribuir a ella. Existe la tendencia práctica de que los procesos de desarrollo (responsabilidad social, gestión ambiental, proyectos de desarrollo, etc.) sean solo responsabilidad del área que los lidera. Sin embargo, el área responsable necesita el apoyo de otras áreas como finanzas, logística, relaciones públicas, comunicaciones, alta gerencia, etc., para lograr sus resultados. Por ello, parte del trabajo que realiza el área líder es comprometer a estas áreas para que aporten en el proceso que se está llevando a cabo.

Todos los aspectos mencionados en este acápite forman un modelo o lista de chequeo de capacidades personales e institucionales que una institución que promueve procesos de desarrollo debe generar para asegurar la sostenibilidad de sus acciones. Se cree que solo invirtiendo en la acción principal el desarrollo será sostenible, cuando, en realidad, hay otros aspectos que deben tenerse en cuenta para lograrlo.

Esta lista también muestra que generar desarrollo no se limita a las capacidades económicas. Y todas estas capacidades no se dan por añadidura a una inversión, sino que es necesario trabajar en ellas. Sin tener en cuenta todas estas capacidades, como lista mínima, existe la probabilidad de que el desarrollo que se busque generar no sea sostenible.

\section{Conclusiones}

Las discusiones sobre crecimiento y desarrollo económico son de larga data entre los intelectuales del campo económico. No cabe duda de que el desarrollo no solo significa mayores ingresos o más producción, sino mejor calidad de vida. En esta discusión, el concepto de desarrollo sostenible aporta un llamado de atención sobre la necesidad de tener una mirada integral del desarrollo, incorporando el eje ambiental. $\mathrm{Y}$ es en este escenario que la necesidad de generar capacidades humanas e institucionales surge como indispensable para la sostenibilidad del desarrollo.

Sin capacidades adecuadas será imposible que los procesos de desarrollo puedan lograr que sus resultados perduren en el tiempo y sirvan de base para procesos cada vez mejores. Contrariamente a lo que se estila en la práctica en países como el Perú, estas capacidades no son solo de inversión o de capital, son de conocimientos, organización, trabajo conjunto y respeto al medio ambiente. En resumen, estas capacidades deben lograr que las personas no solo trabajen en forma conjunta y armoniosa, sino que puedan aprovechar al máximo los recursos con los que cuentan para generar desarrollo. Sin capacidades adecuadas, no habrá desarrollo posible. 


\section{Referencias bibliográficas}

ALONSO, José Antonio. 2004. El desarrollo como proceso abierto al aprendizaje. The European Journal of Development Research. Winter, vol.16, n. ${ }^{\circ}$ 4, págs. 845-867.

COMISIÓN MUNDIAL DEL MEDIO AMBIENTE Y EL DESARROLLO. 1988. Nuestro futuro común. Madrid: Alianza Editorial.

CONGRESO DE LA REPÚBLICA DEL PERÚ. 2007. Ley 29142: Ley de Presupuesto Público 2008, Anexo 5. Lima: MEF.

DE SOTO, Hernando. 2000. El misterio del capital. Lima: El Comercio.

FONDO MONETARIO INTERNACIONAL (FMI). 2002. La función del fortalecimiento de las capacidades en la lucha contra la pobreza. <http://www.imf.org/external/np/ exr/ib/2002/es1/031402s.htm> (Marzo).

FORNI, Pablo et. ál. 2004. ¿Qué es capital social y cómo analizarlo en contexto de exclusión y pobreza? East Lansing, MI: Samora Research Institute, Michigan State University. Research Report N. ${ }^{\circ} 35$.

FURIO BLASCO, E. 2005. Los lenguajes de la economía. 2005. <www.eumed.net/ libros/2005/efb/>.

GALLOPIN, Gilberto. 2006. Los indicadores de desarrollo sostenible: aspectos conceptuales y metodológicos. Ponencia para el Seminario de Expertos sobre Indicadores de Sostenibilidad. Santiago de Chile (agosto).

GARCÍA, Alan. 2007. El síndrome del perro del hortelano. Lima, El Comercio, 28 de octubre.

MALTHUS, Thomas. [1798]. Ensayo sobre el principio de la población. <http://www. eumed.net/cursecon/economistas/malthus. $\mathrm{htm}>$.
MARX, Karl. 1977 [1867]. El capital. 5ºd. México, D. F.: Siglo XXI. Tomo I, vol. 1.

- [1859]. Prólogo a la contribución a la crítica de la economía política. <http://www. eumed.net/cursecon/economistas/marx. $\mathrm{htm}>$.

MORIN, Edgar. 1999. Los siete saberes necesarios para la educación del futuro. Traducción de Nelson Vallejo-Gómez. París: Unesco.

PERROUX, Francois. 1984. El desarrollo y la nueva concepción de la dinámica económica. Barcelona: Serbal / Unesco.

PERÚ. CONSEJO NACIONAL DELAMBIENTE (Conam). 2004. Guía para trabajar el tema ambiental como tema transversal en el aula. Lima: Conam.

PERÚ. INSTITUTO NACIONAL DE ESTADÍSTICA E INFORMÁTICA (INEI). Boletines Informativos $<$ www.inei.gob.pe $>$. Años 2005-2007.

PROGRAMA DE NACIONES UNIDAS PARA EL DESARROLLO (PNUD). 2007. Buenos Aires. <http://www.desarrollohumano.org. ar/Glosario.asp $>$.

PUTNAM, Robert. 1993. Making democracy work. Princenton NJ: Princenton University Press.

RICARDO, David [1817]. 1959. Principios de economía política y tributación. México, D. F.: Fondo de Cultura de Económica.

ROBISON, Lindon et ál. 2000. Social capital and household distributions: Evidence from Michigan and Illinois. East Lansing, MI: Michigan State University. Social Capital Initiative Research Report N. ${ }^{\circ} 12$. 
ROWNTREE, Sebastian [1901]. Poverty: A study of town life. <http://www.spartacus. schoolnet.co.uk/REowntreeS.htm>.

SCHUMACHER. Ernst. 1978. Lo pequeño es hermoso. Madrid: Blume.

SCHUMPETER, Joseph. 2000 [1908-1909]. On the concept of social value. Quarterly Journal of Economics. Vol. 23, págs. 213-232.

SEN, Amartya. 1998. Las teorías de desarrollo a principios del siglo XXI. Cuadernos de Economía. Santa Fe de Bogotá, vol. XVII, n. ${ }^{\circ} 29$, págs. $73-100$.

1992. Capacidad y bienestar. <http:// www.eumed.net/cursecon/economistas/ textos/Sen-capacidad_y_bienestar.htm>.

SMITH, Adam. 1997 [1776]. Investigación sobre la naturaleza y causas de la riqueza de las naciones. México, D. F.: Fondo de Cultura Económica.

SOCIEDAD NACIONAL DE MINERÍA PETRÓLEO Y ENERGÍA (SNMPE). Boletines informativos, reportes mensuales y trimestrales. Lima. <www.snmpe.org.pe>.

SOLANO, David. 2007. Transversalidady transectorialidad en el sector público. Caracas: Centro Latinoamericano de Administración del Desarrollo (CLAD). Mención Honrosa en el Concurso de 2007.

TODARO, Michael P. 1988. El significado del desarrollo y sus diversas teorías explicativas. En: El desarrollo económico del Tercer Mundo. Madrid: Alianza Universidad Textos, págs. 93-140. 